University of Texas Rio Grande Valley

ScholarWorks @ UTRGV

Physics and Astronomy Faculty Publications and Presentations

7-20-2007

\title{
On the age and metallicity estimation of spiral galaxies using optical and near-infrared photometry
}

Hyun Chul Lee

Guy Worthey

Scott C. Trager

S. M. Faber

Follow this and additional works at: https://scholarworks.utrgv.edu/pa_fac

Part of the Astrophysics and Astronomy Commons

\section{Recommended Citation}

Hyun Chul Lee, et. al., (2007) On the age and metallicity estimation of spiral galaxies using optical and near-infrared photometry.Astrophysical Journal664:1 I215. DOI: http://doi.org/10.1086/518855

This Article is brought to you for free and open access by the College of Sciences at ScholarWorks @ UTRGV. It has been accepted for inclusion in Physics and Astronomy Faculty Publications and Presentations by an authorized administrator of ScholarWorks @ UTRGV. For more information, please contact justin.white@utrgv.edu, william.flores01@utrgv.edu. 


\title{
ON THE AGE AND METALLICITY ESTIMATION OF SPIRAL GALAXIES USING OPTICAL AND NEAR-INFRARED PHOTOMETRY
}

\author{
Hyun-chul LeE and GuY Worthey \\ Department of Physics and Astronomy, Washington State University, Pullman, WA 99164-2814
}

Scott C. Trager

Kapteyn Astronomical Institute, University of Groningen, Postbus 800, NL-9700 AV Groningen, Netherlands

AND

S. M. FABER

UCO/Lick Observatory and Department of Astronomy and Astrophysics, University of California Santa Cruz, Santa Cruz, CA 91101 Received 2006 May 17; accepted 2007 April 12

\begin{abstract}
In integrated light, some color-color diagrams that use optical and near-infrared photometry show surprisingly orthogonal grids as age and metallicity are varied, and they are coming into common usage for estimating the average age and metallicity of spiral galaxies. In this paper we reconstruct these composite grids using simple stellar population models from several different groups convolved with some plausible functional forms of star formation histories at fixed metallicity. We find that the youngest populations present $(t<2 \mathrm{Gyr})$ dominate the light, and because of their presence the age-metallicity degeneracy can be partially broken with broadband colors, unlike older populations. The scatter among simple stellar population models by different authors is, however, large at ages $t<2$ Gyr. The dominant uncertainties in stellar population models arise from convective core overshoot assumptions and the treatment of the thermally pulsing asymptotic giant branch phase and helium abundance may play a significant role at higher metallicities. Real spiral galaxies are unlikely to have smooth, exponential star formation histories, and burstiness will cause a partial reversion to the single-burst case, which has even larger model-to-model scatter. Finally, it is emphasized that the current composite stellar population models need some implementation of chemical enrichment histories for the proper analysis of the observational data.
\end{abstract}

Subject headings: galaxies: abundances — galaxies: evolution — galaxies: photometry — galaxies: spiral — galaxies: stellar content

Online material: color figures

\section{INTRODUCTION}

Many recent works on age estimation of spiral galaxies have used the surprisingly orthogonal color-color grids that combine optical and near-infrared colors (e.g., Bell \& de Jong 2000; MacArthur et al. 2004). The "surprise" comes from a long list of previous works starting with Worthey (1994) that showed that age effects and metallicity effects in old stellar populations are very similar on colors, i.e., the age-metallicity degeneracy problem. Age and metallicity vectors projected onto color-color diagrams from simple stellar populations are not orthogonal, but lie nearly parallel to each other so that age effects and metallicity effects are nigh indistinguishable. The purpose of this study is to investigate whether the new, seemingly successful method to break the agemetallicity degeneracy is convincingly robust or not. The benefits of near-IR photometry combined with optical photometry have been addressed by many groups and applied to wide variety of stellar systems (e.g., de Jong 1996; Puzia et al. 2002; MacArthur et al. 2004; Larsen et al. 2005; Kundu et al. 2005; James et al. 2006). The basic claim is that age-sensitive main-sequence turnoffs (MSTOs) dominate the visible band, while metallicity-sensitive giant branches dominate the near-IR, and that the photometric behavior of these two evolutionary regions is sufficiently different to provide diagnostic power in terms of deriving average ages and metallicities.

It is important to note, however, that the orthogonal grids that appear in the literature are not simple stellar population (SSP) models, but composite stellar population (CSP) ones. The CSP models that we study here are different from the SSP ones in that CSP models are made by convolving SSPs with some plausible star formation histories (SFHs) at fixed metallicity and fixed initial mass function. Obviously, SSP models that consist of a single age and a single metallicity are not good representations of the protracted and ongoing SFH of spiral galaxies.

Before we reconstruct and assess the composite models, however, we investigate the SSP models in detail because they are the building blocks of the composite models. We will compare several different groups' SSP models that have become available lately and look into their similarities and differences. In particular, we examine the robustness of stellar population models at ages younger than 5 Gyr because we perceive that these younger ages dominate the behavior of the composite models because of their overwhelming luminosity.

We then adopt an exponentially declining star formation rate (often called a $\tau$ model — see Table 1) in order to make composite models. We make note of the relationship between SSP and CSP models and what information flows from the simple to the complex. Other issues, such as different chemical enrichment histories, helium abundance, and possible variations of initial mass function (IMF) as a function of metallicity are briefly addressed.

\section{STELLAR POPULATION MODELS}

Many integrated-light models are now available for those interested in interpreting galaxy spectra and colors. Since we are starting with the observational studies of Bell \& de Jong (2000) 


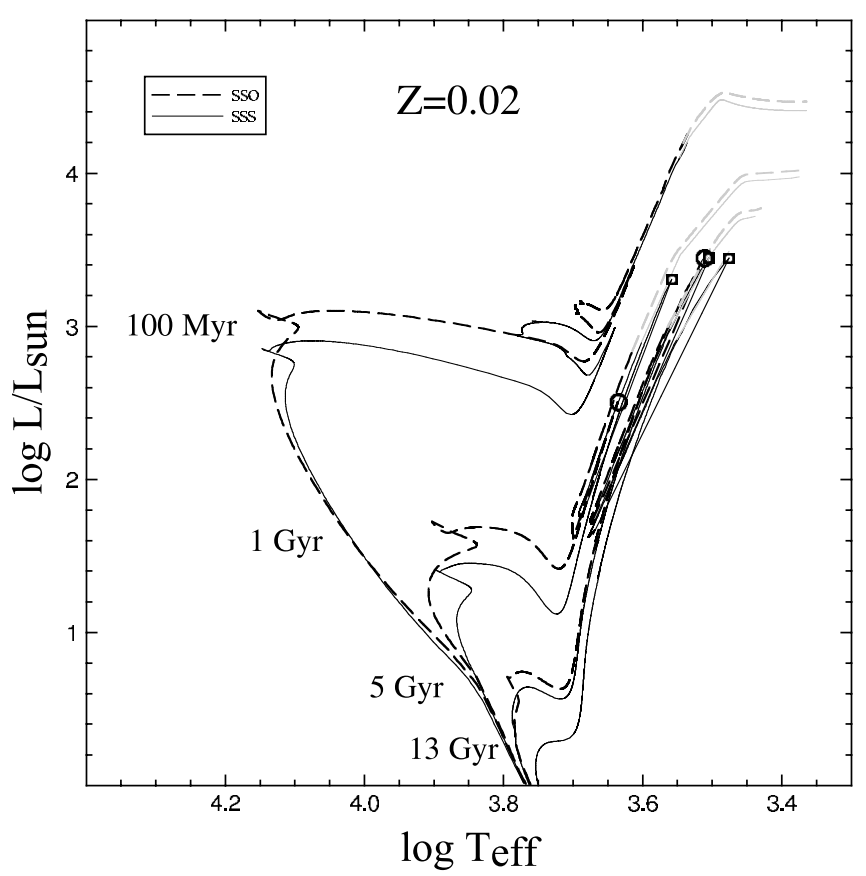

FIG. 1.- Teramo isochrones (BaSTI) are illustrated at 0.1, 1, 5, and $13 \mathrm{Gyr}$ at solar metallicity. Those labeled sso are solar-scaled isochrones with convective core overshoot effects (dashed lines), while the sss are solar-scaled isochrones without overshooting effects (solid lines). The RGB tips are marked by squares (without overshooting) and circles (with overshooting) at 1, 5, and 13 Gyr. Gray lines: Full extension along the TPAGB phases of the BaSTI. [See the electronic edition of the Journal for a color version of this figure.]

and MacArthur et al. (2004), we certainly must include the stellar evolution on which the models of Bruzual \& Charlot (2003, hereafter BC03) are based because those are the models adopted by MacArthur and Bell to build their diagnostic CSP models, assuming an exponentially declining SFH at fixed IMF and fixed metallicity. The rather minor impact of adopting different forms of the IMF (e.g., Salpeter 1955; Kroupa 2002; Chabrier 2003) is convincingly demonstrated in MacArthur et al. (2004), so we will not explore IMF variation in any depth, but will stick with a Salpeter (1955) IMF. Besides BC03 models, ${ }^{1}$ we will also examine Raimondo et al. (2005; the Teramo group's SPoT ${ }^{2}$ ), and Maraston (2005, hereafter M05). ${ }^{3}$

Both HR diagram behavior and SSP photometric models will be examined before we look into CSP models. Of key interest are the effects from convective core overshooting and from the thermally pulsing asymptotic giant branch phase at relatively young ages (100 Myr to $2 \mathrm{Gyr}$ ), so we wish to include a variety of different approaches to these aspects of evolution theory.

To do this, we use photometric models that are direct descendents of an evolutionary population synthesis code that has been developed to study the stellar populations of star clusters and galaxies (Lee et al. 2002, 2004) that includes as its evolutionary basis the $Y^{2}$ isochrones (Yi et al. 2001). However, now that we are extending down to $100 \mathrm{Myr}$, the $Y^{2}$ isochrones may not be the best choice for this study because there are no post-RGB stellar models yet for the younger ages. We therefore include the Teramo groups' isochrones, $\mathrm{BaSTI}^{4}$ (Pietrinferni et al. 2004; Cordier et al.

\footnotetext{
1 See http://www.cida.ve/ bruzual/bc2003 (based on Padova 1994 isochrones).

2 See http://193.204.1.79:21075 (based on the earlier version of Teramo isochrones).

3 See http://www-astro.physics.ox.ac.uk/ maraston.

4 See http://www.te.astro.it/BASTI/index.php (incorporating the last modification of 2006 March 24).
}

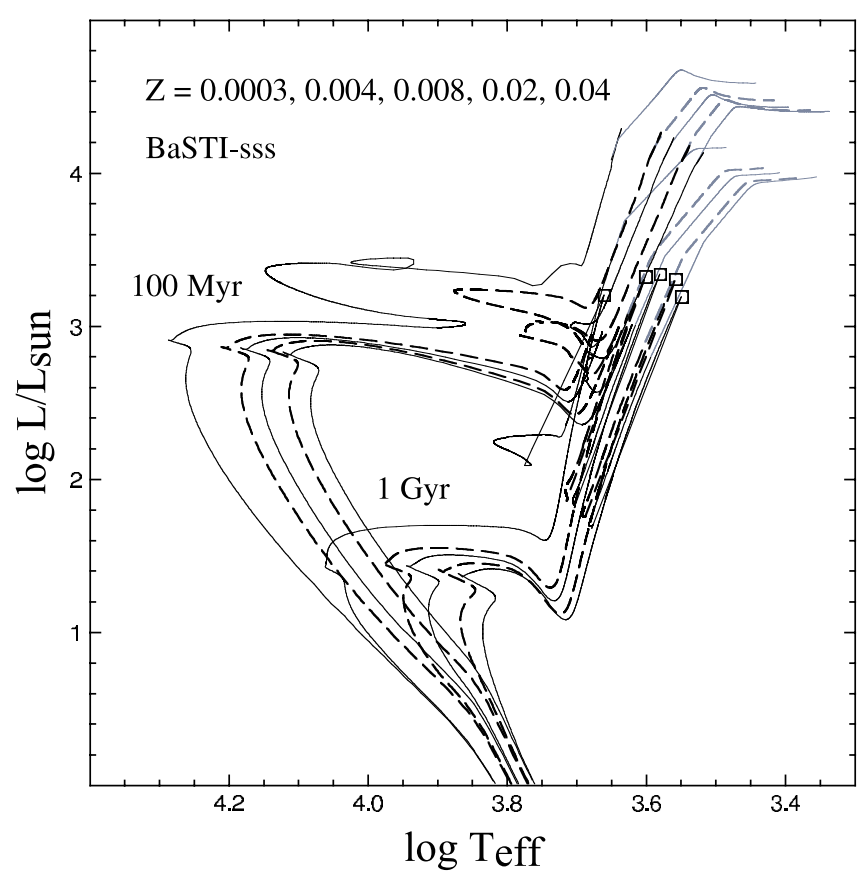

FIG. 2.-BaSTI-sss (solar-scaled standard; no overshooting) isochrones are compared at $100 \mathrm{Myr}$ and at $1 \mathrm{Gyr}$ for the five metallicities that we illuminate in this study (Figs. 4, 5, 10, and 11). To guide the eye, $Z=0.004$ and $Z=0.02$ are drawn with dashed lines. Also, the RGB tips are marked by squares at $1 \mathrm{Gyr}$. Note that the blue loop phase at solar metallicity locates at the hotter side compared to that of $Z=0.008$ at $100 \mathrm{Myr}$ in these isochrone sets. Gray lines: Full extension along the TPAGB phases of the BaSTI. [See the electronic edition of the Journal for a color version of this figure.]

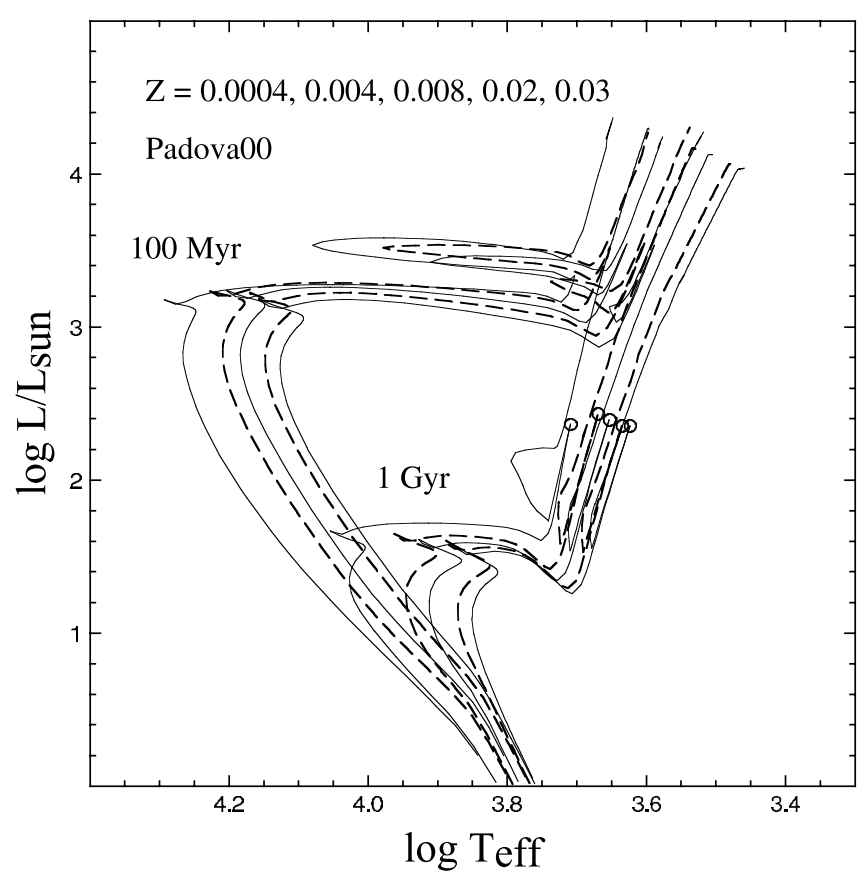

FIG. 3.-Similar to Fig. 2, but Padova00 isochrones are displayed at $100 \mathrm{Myr}$ and at $1 \mathrm{Gyr}$ for the five metallicities. To guide the eye, $Z=0.004$ and $Z=0.02$ are drawn with dashed lines. The RGB tips are marked by circles where they exist (at $1 \mathrm{Gyr}$ ), but they do not go all the way to $\log L \approx 3.2$ due to convective core overshoot effects. Note also that this isochrone sets are relatively brighter than the ones in Fig. 2 because of overshooting. Moreover, the blue loop phases at $100 \mathrm{Myr}$ are systematically shifting to the right with increasing metallicity compared to the ones in Fig. 2. [See the electronic edition of the Journal for a color version of this figure.] 
2007), and the Girardi et al. (2000) isochrones in this study. We investigate ages from $100 \mathrm{Myr}$ to $13 \mathrm{Gyr}$ and metallicities from $Z=0.0003$ to 0.05 . We adopt a $100 \mathrm{Myr}$ limit for the most recent epoch of star formation in this study in order to accommodate and compare as many different stellar population models as possible. $^{5}$

\subsection{HR Diagrams}

\subsubsection{Effects Due to Convective Core Overshooting}

We study effects due to convective core overshooting here. Stars more massive than $\sim 1 M_{\odot}$, depending on the chemical composition, are known to develop a convective core during their hydrogenburning phases as a result of the strong dependence of $\mathrm{CNO}$ cycle energy generation on temperature. The convective motion in the core could go beyond the physical Schwarzschild boundary, carried by momentum. In principle, this overshooting phenomenon would make a larger helium core, a brighter luminosity, and a longer lifetime because of the increased fuel supply. The chemical composition of the stars determines the exact transition mass of convective core overshoot (e.g., Demarque et al. 2004; VandenBerg et al. 2006). Stellar rotation may affect it as well (Deupree 1998, 2000; Meynet \& Maeder 2000). For instance, Girardi et al. (2000) adopt $\sim 1 M_{\odot}$ as the transition mass independent of chemical composition, even though they gradually change overshooting efficiency in the mass range of $1.0 M_{\odot}<M<$ $1.5 M_{\odot}$. The comparisons of transition mass treatment among several different groups are well described in Gallart et al. (2005, supplement section, Fig. 4).

Figure 1 demonstrates the effects due to convective core overshoot from the BaSTI. In this HR diagram, stellar isochrones with and without overshooting effects are depicted at ages $0.1,1$, 5, and $13 \mathrm{Gyr}$ at solar metallicity. The tracks labeled sss are solarscaled standard isochrones without convective core overshoot and those labeled sso are solar-scaled isochrones with overshooting effects. ${ }^{6}$ It is clearly seen in Figure 1 that stars with convective core overshoot (dashed lines) are generally more luminous and show less-extended blue loop phase at $100 \mathrm{Myr}$ than stars without this phenomenon. Moreover, Figure 1 shows that overshooting effects are more conspicuous at $1 \mathrm{Gyr}$ than at $100 \mathrm{Myr}$, especially near the MSTO region in the case of the BaSTI isochrones. These dissimilarities will be converted and displayed as integrated-light color differences in Figures 4 and 5.

It is also interesting to find in Figure 1 that the overshooting effects persist even at $5 \mathrm{Gyr}$ for solar metallicity in a minor way. However, overshooting effects should disappear altogether at around $5 \mathrm{Gyr}$ for the metal-poor stars as they become less massive compared to those of solar metallicity at the same age. Pietrinferni et al. (2004) note that, for ages less than $\sim 4 \mathrm{Gyr}$, models including overshooting provide a "better match" to the color-magnitude diagrams of star clusters in the Milky Way Galaxy. As stars become less massive, however, they develop radiative cores and the issue of overshooting effect eventually vanishes, so convective core overshooting is only important at ages $t<5 \mathrm{Gyr}^{7}$

\footnotetext{
${ }^{5}$ Although BC03 and M05 give their stellar population models starting from $1 \mathrm{Myr}, \mathrm{BaSTI}$, for example, present their stellar models starting from $\sim 30 \mathrm{Myr}$. We also note that there are many uncertainties such as rotation, mass loss, and magnetic activity, to name a few, for the fine constraints of massive star evolution as well as its following supergiant evolutionary stage (e.g., Meynet et al. 2007).

${ }^{6} \mathrm{BaSTI}$ employs $1.1 M_{\odot}$ as the transition mass and change the overshooting efficiency in the mass range of $1.1 M_{\odot}<M<1.7 M_{\odot}$.

${ }^{7}$ In fact, BaSTI does not provide old $(t>10 \mathrm{Gyr})$ stellar models with overshooting effects because there should not be any differences.
}

\subsubsection{Effects Due to the Treatment of TPAGB Stars}

We now investigate the effects of the thermally pulsing asymptotic giant branch (TPAGB) phase. The TPAGB phase is the last stage of AGB evolution. In general, the AGB stars start to appear at $\sim 50 \mathrm{Myr}$, while RGB stars emerge around $2 \mathrm{Gyr}$ (Gallart et al. 2005; Raimondo et al. 2005; Mucciarelli et al. 2006). Therefore, after the high-mass MS stars and supergiants fade away, it is AGB stars that dominate the integrated bolometric light until fullfledged RGB stars are looming. The lifetime of AGB stars depends on their mass and composition but is of the order of $10^{7} \mathrm{yr}$, with about $90 \%$ of the time spent on the early-AGB phase and the rest on the TPAGB. The TPAGB phase dominates the light in the near-IR at young ages ( $t<2 \mathrm{Gyr}$ ), even though the number of stars in this phase is small compared with the number of stars in earlier phases. After $t>2 \mathrm{Gyr}$, the RGB tip becomes nearly as bright as the AGB tip but is much more numerously populated, by nearly a factor of 10 (Ferraro et al. 2004). In Figure 1 (and in Fig. 2), the TPAGB phases are depicted with gray lines, as described in the BaSTI Web site. ${ }^{8}$

Because of the well-known core mass-luminosity relation of AGB stars (Paczyński 1971), it is shown in Figure 1 that the luminosity of TPAGB stars becomes dimmer as the population becomes older, i.e., the stellar masses decrease. Therefore as Figure 1 graphically illuminates, the TPAGB stars are most important at younger ages, not only because they are brighter at high mass but also because RGB stars do not reach the typical RGB tip luminosity since their cores are not degenerate. However, once stars go all the way to the RGB tip and suffer a helium flash, as the population ages (as their masses become lighter and their cores become degenerate) it is RGB stars instead of AGB stars that dominate the near-IR flux.

Recently, several different groups have investigated this interesting but complex set of stellar evolutionary phases. Vassiliadis \& Wood (1993), for instance, predicted that the bolometric luminosity of the tip of the AGB should remain much brighter than the tip of the RGB. As a matter of fact, the brightest stars in some star clusters in the LMC and SMC, SWB ${ }^{9}$ IV-VI, are carbon-rich AGB stars, and they are generally about $1 \mathrm{mag}$ brighter in $K$ band than the tip of the RGB (Mould \& Aaronson 1979, 1980; Frogel et al. 1980; Cohen et al. 1981; Ferraro et al. 2004; Mucciarelli et al. 2006). Because of carbon-rich AGB stars, the near-IR colors of these star clusters are much redder than those of older or younger clusters, whereas the optical colors redden more monotonically with cluster age.

In order to incorporate these observational facts, $\mathrm{BC} 03$, for example, adopted Vassiliadis \& Wood (1993) values to extend beyond the early-AGB and took Groenewegen \& de Jong (1993) and Groenewegen et al. (1995) values for the relative lifetimes of carbon-rich TPAGB stars. Recently, Marigo et al. (2003) have successfully reproduced the 2MASS and DENIS observations of the red tail of carbon-AGB stars in the LMC theoretically in the $(J-K, K)$ diagram using carbon-rich opacities instead of the oxygen-rich opacities of $\mathrm{M}$ giants. They indeed recognize that carbon stars are about 1 mag brighter in $K$ band than oxygen-rich M stars. Historically, it was Blanco et al. (1978) who first found that the number ratio of $\mathrm{C} / \mathrm{M}$ stars decreases sharply with increasing

\footnotetext{
${ }^{8}$ An additional 250 points for the TPAGB phases on top of the already existing 2000 points were recently appended to the Teramo isochrones and are available at the BaSTI Web site (Cordier et al. 2007).

9 The Searle et al. (1980) classification scheme for star clusters in the Magellanic Clouds. In general, it is interpreted that star clusters with type I are the youngest and that those with type VII are the oldest.
} 


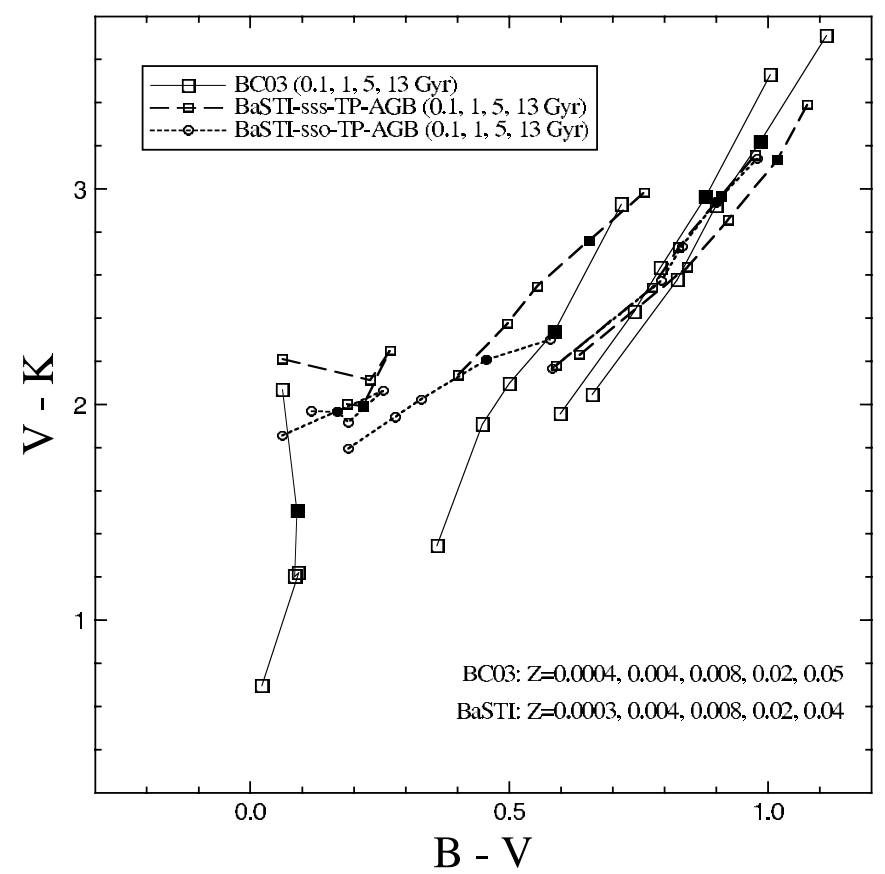

Fig. 4.-A $(B-V)$ vs. $(V-K)$ plot of simple stellar population models at $0.1,1,5$, and 13 Gyr. Lines connect different metallicities at given ages. Solid lines with big open squares: $\mathrm{BC} 03$ (also shown in Figs. 5, 6, and 7). Dashed lines with small open squares: Our computations using BaSTI-sss isochrones including TPAGB phases. Dotted lines with open circles: Those using BaSTI-sso isochrones. At 13 Gyr, only the BaSTI-sss case is shown because there is no overshooting effect at $13 \mathrm{Gyr}$. To guide the eye, solar metallicity models are marked by filled symbols. [See the electronic edition of the Journal for a color version of this figure.]

metallicity from the SMC, to the LMC, to the Galactic Bulge. This is because stars with lower metallicities require fewer thermal pulses to change from O-rich to C-rich stars (Liu et al. 2000).

Figures 2 and 3 compare BaSTI and Padova isochrones at $100 \mathrm{Myr}$ and at $1 \mathrm{Gyr}$ for five different metallicities. Figure 2 shows the solar-scaled, standard (no overshooting) BaSTI, while Figure 3 depicts the Padova 2000 isochrones (Girardi et al. 2000; hereafter Padova00) that adopt overshooting effects as a default. There are subtle differences between Figures 2 and 3, mostly because of the different input physics ${ }^{10}$ that go into each stellar model. First, contrary to the BaSTI-sss isochrones, at $100 \mathrm{Myr}$ the Padova00 isochrones become systematically redder with increasing metallicity, particularly in the blue loop phases. This results in the wide range in $V-K$ color as a function of metallicity in the simple stellar population models based on Padova isochrones, such as $\mathrm{BC} 03 .{ }^{11}$ Second, compared to the recent description of full extension along the TPAGB phases of the BaSTI (Fig. 2, gray lines), we deduce that the Padova00 isochrones do not have the TPAGB phase at $100 \mathrm{Myr}$, but at $1 \mathrm{Gyr}$ they go all the way to the TPAGB luminosity, although they seem to fail to illuminate carbon stars, as Marigo et al. (2003) pointed out. It is also worthwhile to reiterate that Padova00 employs convective core overshooting as a default, and that this makes them, on the whole, brighter than BaSTI-sss. Moreover, at 1 Gyr the RGBs of Padova00 do not go all the way to the tip luminosity because of the overshooting effect, as illustrated in Figure 1.

\footnotetext{
${ }^{10}$ Including different choices of $\Delta Y / \Delta Z($ BaSTI $=1.4$, Padova00 $=2.25)$.

11 BC03 prefer Padova 1994 isochrones (Bertelli et al. 1994; hereafter Padova94) to Padova 2000 ones (Girardi et al. 2000) because the former better matches galaxy colors with relatively low giant branch temperatures. Otherwise, shapes and locations of these two Padova isochrones are extensively similar in the HR diagram.
}

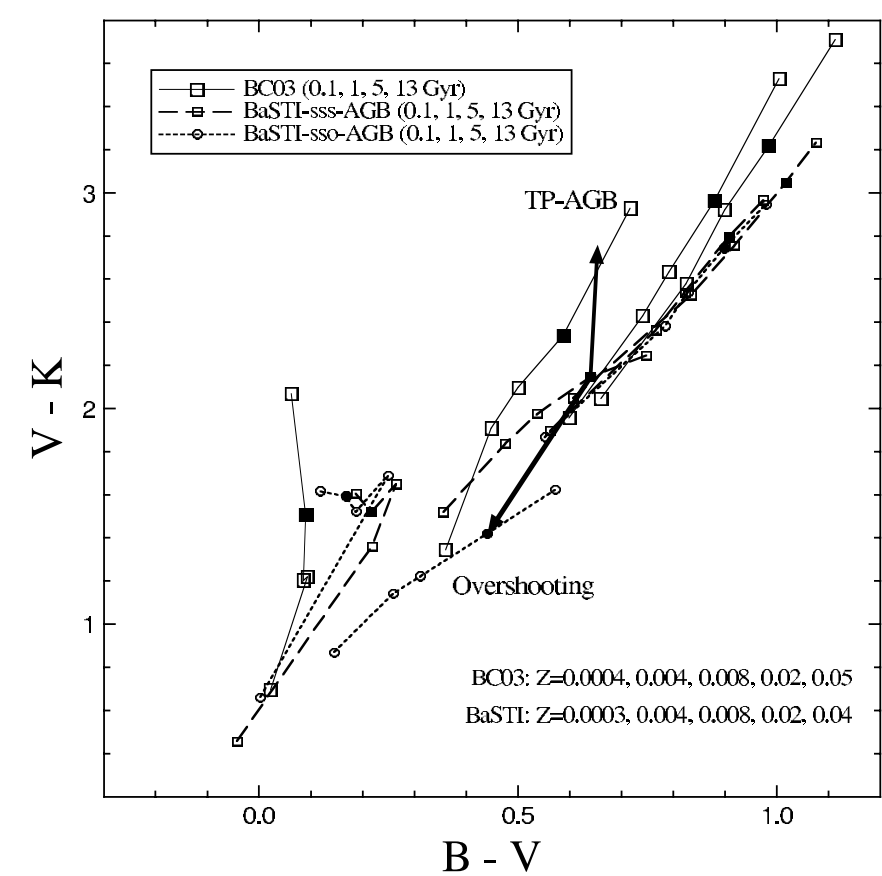

FIG. 5.- Same as Fig. 4, but our computations using the BaSTI-sss and BaSTI-sso isochrones without TPAGB phases are shown as dashed and dotted lines, respectively. At 1 Gyr and solar metallicity, for the BaSTI-sss isochrones, the TPAGB and the overshooting effects are depicted with vectors. [See the electronic edition of the Journal for a color version of this figure.]

\subsection{Color-Color Diagrams}

Having examined the key uncertainties of stellar models at young ages in the HR diagram, we now compare SSP models in color-color diagram. The $B-V$ versus $V-K$ diagrams will be examined first. This is because there are well-established observational data sets of star clusters and galaxies in $B V K$ passbands. Later we will switch to the familiar $B-R$ versus $R-K$ plots that Bell \& de Jong (2000) and MacArthur et al. (2004) presented in their papers.

Figures 4-7 show several different SSP models at $0.1,1,5$, and $13 \mathrm{Gyr}$ in the $B-V$ versus $V-K$ diagram. At a given age, five metallicities are delineated as indicated at the bottom right. To guide the eye, solar metallicities are marked by filled symbols at given ages. The solid lines with big open squares are from $\mathrm{BC} 03$.

In Figures 4 and 5, our SSP computations using the BaSTI with and without convective core overshoot are separately plotted at $0.1,1$, and 5 Gyr of age. ${ }^{12}$ Dashed lines with open squares are our models using BaSTI-sss and dotted lines with open circles are BaSTI-sso. The only difference between Figures 4 and 5 is in our SSP computations: we adopt the BaSTI all the way to the TPAGB in Figure 4, while Figure 5 shows the effect of cutting out the TPAGB phases altogether. In this way, we can see the size of the color shifts arising from the TPAGB contribution (e.g., Figs.1 and 2, gray lines).

The effects due to convective core overshoot are also displayed in Figures 4 and 5. They are dominant, as expected, at young ages only, here exemplified at $100 \mathrm{Myr}$ and at 1 Gyr. In particular, the differences due to overshooting are more outstanding at $1 \mathrm{Gyr}$ than $100 \mathrm{Myr}$, as expected from the HR diagram from the BaSTI in Figure 1. Moreover, metallicity differences from $Z=0.0003$

\footnotetext{
12 Our computations using BaSTI-sss isochrones are also plotted at 13 Gyr. Following the discussion above, isochrones with overshooting will be identical at this age.
} 


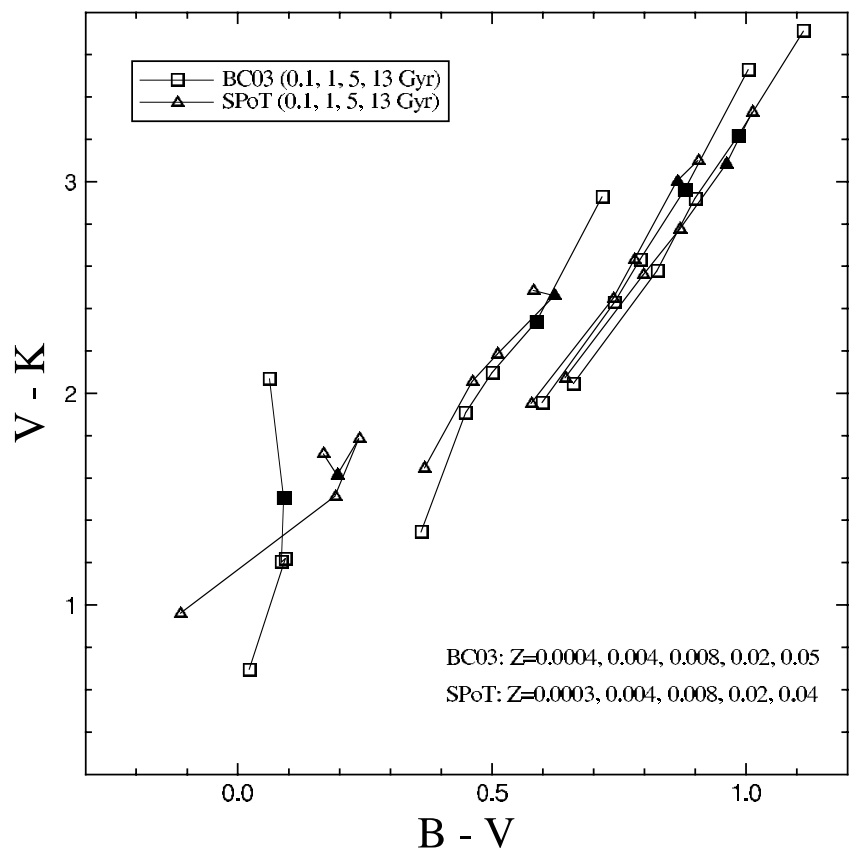

FIG. 6.-Similar to Fig. 4, but the SPoT models (solid lines with open triangles) are compared with $\mathrm{BC} 03$. To guide the eye, solar metallicity models are marked by filled symbols. [See the electronic edition of the Journal for a color version of this figure.]

to $Z=0.004$ at $100 \mathrm{Myr}$ give rise to significant color differences in Figure 5 because the MS turnoffs and blue loops are considerably hotter at $Z=0.0003$ compared to those at $Z=0.004$, as illustrated in Figure 2. Furthermore, it should be noted that at $100 \mathrm{Myr}$ the colors from our computations (also from the SPoT in Fig. 6 and from M05 in Fig. 7) at solar metallicity are bluer compared to those at $Z=0.008$ because the blue loop phase of the BaSTI at solar metallicity is hotter than the $Z=0.008$ blue loop. ${ }^{13}$ It is also interesting to find that only BC03's 100 Myr models cover a wide range in $V-K$ as a function of metallicity: this will later result in the nice orthogonal grids seen in the composite models. By comparing Figures 2 and 3, it can be seen that the continual redward march of the blue loop as a function of metallicity of the Padova isochrones causes BC03's wide spread in $V-K .^{14}$

Comparing Figures 4 and 5, the models lie more closely together in Figure 5, especially at $100 \mathrm{Myr}$, indicating that the BaSTI's TPAGB phases contribute substantial luminosity in the $K$ band. ${ }^{15}$ There are profound color differences caused by convective core overshoot and TPAGB phenomena, especially at 1 Gyr. This can be comprehended by examining the HR diagrams in Figures 1 and 2. At $1 \mathrm{Gyr}$ and solar metallicity, for the BaSTI-sss, the TPAGB phase makes $B-V$ only 0.013 mag redder but $V-K 0.614$ mag redder. Without considering those TPAGB phases, as indicated in Figure 5, the overshooting effects alone make $B-V 0.199$ mag bluer and $V-K 0.727$ mag bluer at $1 \mathrm{Gyr}$ and solar metallicity. They are depicted with vectors in Figure 5. Furthermore, it is important to note that the $\mathrm{BC} 03$ models' highest metallicities go

\footnotetext{
13 The stellar evolutionary phase of the blue loop is notoriously difficult to compute and the metallicity does not seem to be the first controlling parameter that governs that phase (S. Cassisi 2006, private communication).

14 Also, bear in mind that BC03's highest metallicity is Padova94's $Z=0.05$ compared to Padova00's $Z=0.03$ that we see in Fig. 3 .

15 BC03 have recently conceded that their treatment of TPAGB phases needs to be revised (see Bruzual 2007).
}

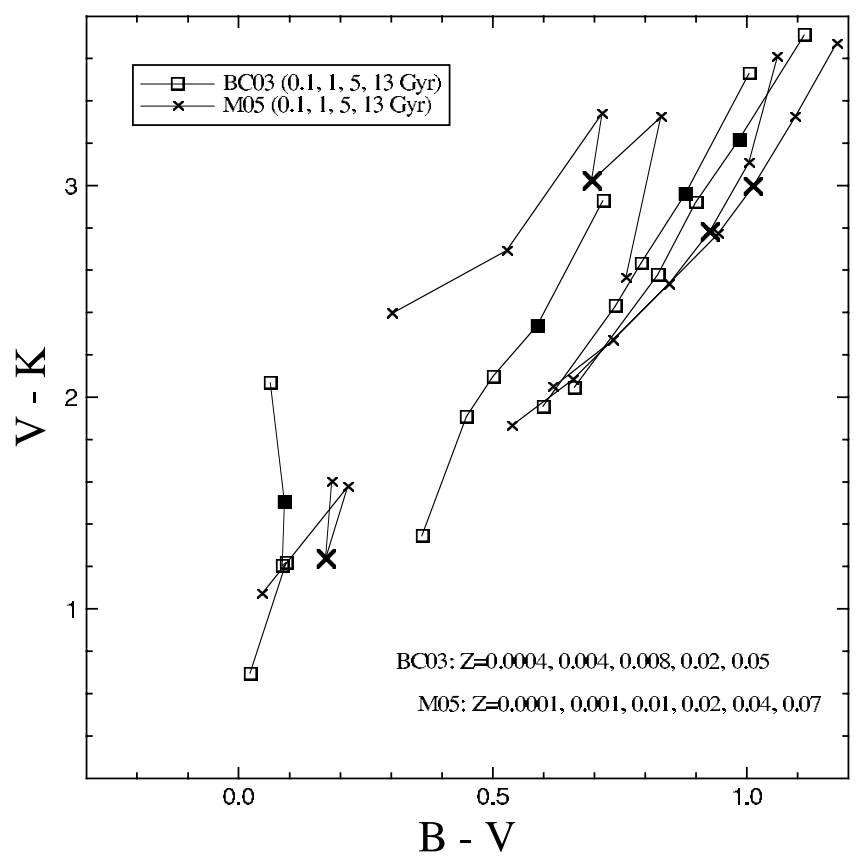

FIG. 7.- Similar to Fig. 6, but the Maraston (2005; M05) models (solid lines with crosses) are compared with $\mathrm{BC} 03$. BC03's and M05's metallicities are indicated at the bottom right. At $100 \mathrm{Myr}, \mathrm{M} 05$ are only given at $Z=0.001,0.01$, 0.02 , and 0.04 . To guide the eye, solar metallicity models are marked by filled or larger symbols. [See the electronic edition of the Journal for a color version of this figure.]

very red in $V-K$ compared to other SSPs at 5 and 13 Gyr. This is possibly because BC03's highest metallicity is $Z=0.05$ with $Y=0.352$ from the Padova94 assumptions, while the others employ the BaSTI at $Z=0.04$ with $Y=0.303$.

In Figure 6, BC03 and SPoT models are compared. The solid lines with open triangles are from Raimondo et al. (2005). ${ }^{16}$ To guide the eye, solar metallicities are marked with filled symbols. By comparing the SPoT models in Figure 6 with our calculations in Figures 4 and 5, which are similarly based on the BaSTI, it seems that SPoT's TPAGB treatment (their B1 models with moderate mass-loss rate) is in between our computations of scenarios with and without the Cordier et al. (2007) TPAGB description. For $\mathrm{BC} 03$, it is also most important to note that at 100 Myr the wide range of metallicities merely generates small variations, less than $0.2 \mathrm{mag}$, in $B-V$. This is understandable from Figure 3, where the hot upper main sequence stars counterbalance the relatively cool blue loop phase stars to make an intriguingly narrow range in integrated $B-V$ color at $100 \mathrm{Myr}$.

BC03 models are compared with M05 models in Figure 7. Solid lines with big open squares are from $\mathrm{BC} 03$, while those with crosses are from M05. To guide the eye, solar metallicities are marked with filled and larger symbols at four ages: $0.1,1,5$, and 13 Gyr. At 100 Myr, M05 models are only given at four metallicities, i.e., $Z=0.001,0.01,0.02$, and 0.04 , so that the bluest color of M05 at $100 \mathrm{Myr}$ is $Z=0.001$. To extend the highmetallicity end, M05 implemented $3.5 Z_{\odot}$ metallicity Padova stellar models from Salasnich et al. (2000) in the fuel-consumption theorem-based models. Here, we show those M05 SSP models that have a blue horizontal branch at the two lowest metallicities. It is

16 The SPoT models were developed before BaSTI isochrones have the full extension along the TPAGB phases. They present four different TPAGB cases depending on mass-loss rates. We show their B1 models with moderate mass-loss rate in this study as posted at the SPoT Web site. 


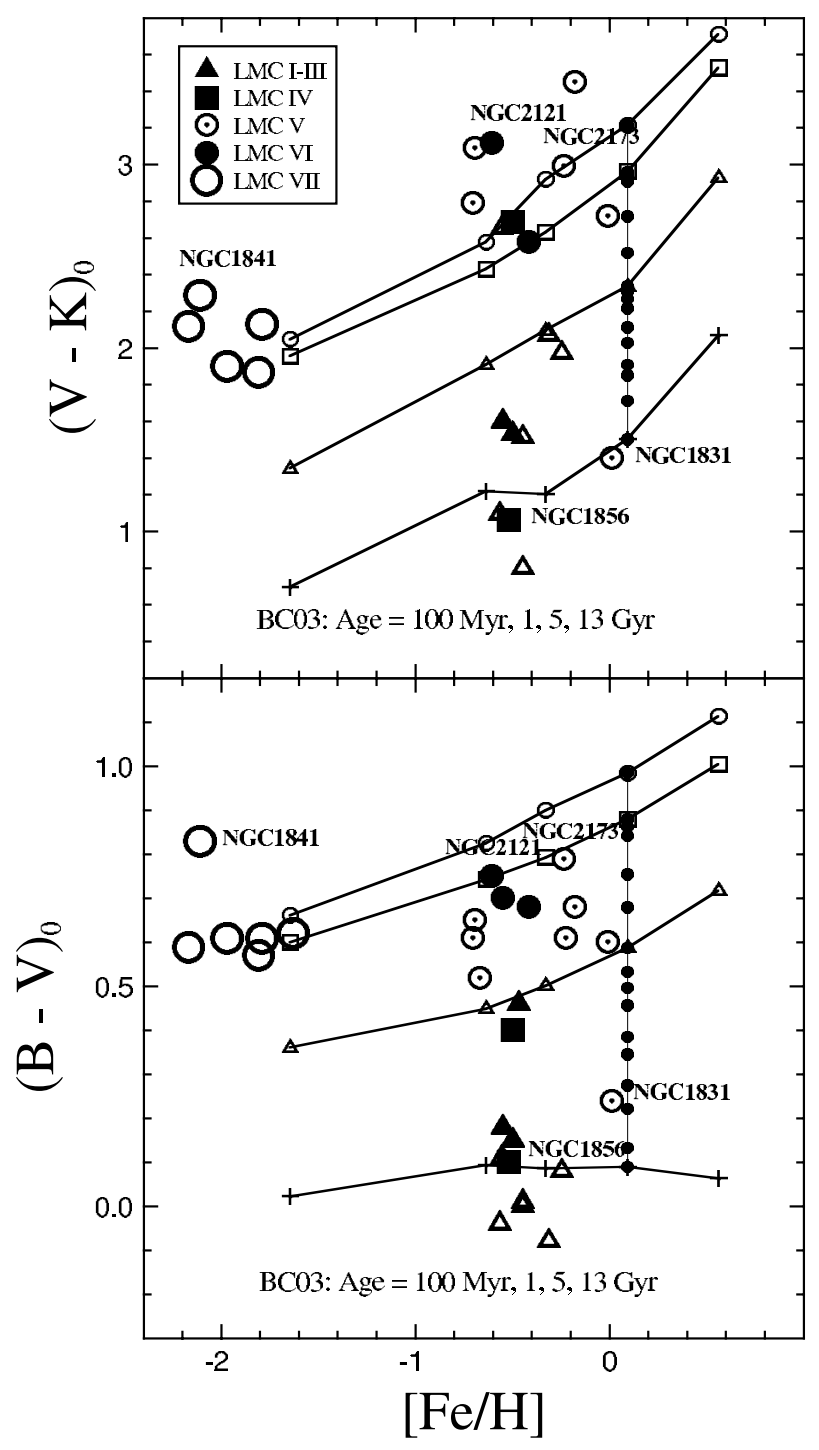

Fig. 8. - LMC star clusters are compared with $\mathrm{BC} 03$ models at $[\mathrm{Fe} / \mathrm{H}]$ vs. $(B-V)_{0}$ (bottom) and $(V-K)_{0}$ (top). Among SWB types I-III, star clusters younger than $100 \mathrm{Myr}$ are depicted with open triangles. At solar metallicity, eight $100 \mathrm{Myr}$ increments between $100 \mathrm{Myr}$ and $1 \mathrm{Gyr}$, and 1.5, 2, 3, and $4 \mathrm{Gyr}$ between 1 and $5 \mathrm{Gyr}$ are connected with dots. Both $(B-V)$ and $(V-K)$ colors of $\mathrm{BC} 03$ models are becoming monotonically redder with increasing age at given metallicity. Note that contrary to $(B-V)$ colors, $(V-K)$ colors show some evident mismatch between intermediate-age LMC star clusters and model predictions. Ages and metallicities of LMC clusters are taken from Pessev et al. (2006). Recent age estimates of NGC 1856 (300 Myr), NGC 1831 (700 Myr), NGC 2173 (1.6 Gyr), and NGC 2121 (2.9 Gyr) are from Kerber et al. (2007). NGC 1841 is indicated because of its unusually red color in $(B-V)_{0}$ (see text). [See the electronic edition of the Journal for a color version of this figure.]

intriguing to note that the most serious differences between two models are manifested at $1 \mathrm{Gyr}$, to a degree similar to (but more significantly discrepant than) what we have seen from the model comparisons in Figures 4 and 5. This is because of different descriptions of both the convective core overshoot and the TPAGB treatment (Maraston et al. 2006).

\section{COMPARISON OF MODELS WITH OBSERVATIONS}

Having discussed the theoretical uncertainties in stellar models and their ramifications on the SSP models, we now present a comparison between theoretical models and observational data. In Figures 8-12, observational data from Large Magellanic Cloud

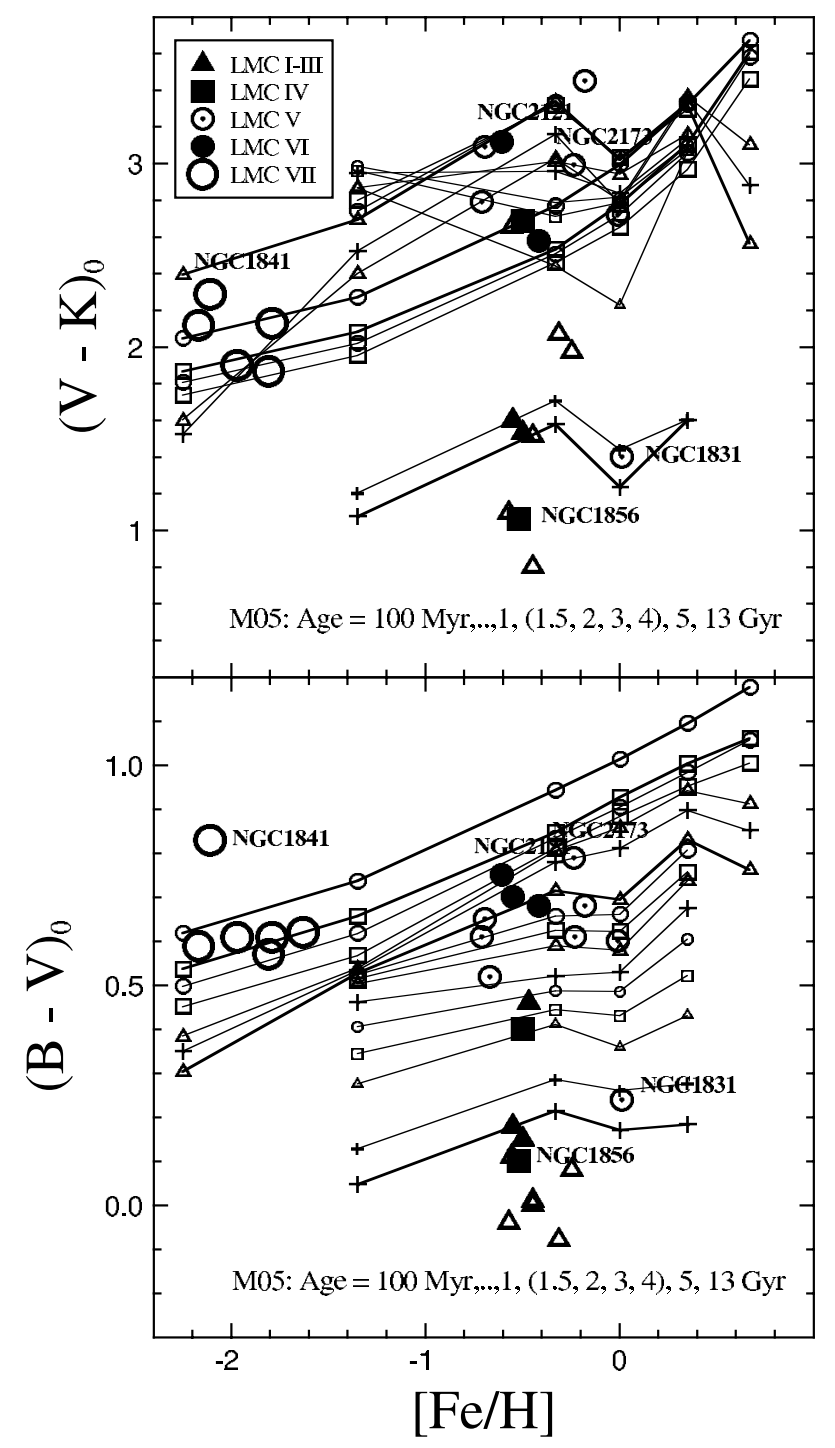

FIG. 9.- Same as Fig. 8, but LMC star clusters are overlaid on M05 models. Four ages $(100 \mathrm{Myr}, 1,5,13 \mathrm{Gyr})$ that are shown in Fig. 7 are delineated with thicker lines with crosses, triangles, squares, and circles, respectively. Between $100 \mathrm{Myr}$ and $1 \mathrm{Gyr}$, every $100 \mathrm{Myr}$ increment is depicted with the following symbol order: cross, triangle, square, and circle. The same symbols are used for age increments between 1 and 5 Gyr. While the $(B-V)$ colors become monotonically redder with increasing age at given metallicity, her $(V-K)$ colors show a sudden leap at 300 Myr because of the onset of the TPAGB. M05 tuned her fuel-consumption theorem parameters using the older age estimation of intermediate-age LMC star clusters. Recent age estimates of NGC 1856 (300 Myr), NGC 1831 (700 Myr), NGC 2173 (1.6 Gyr), and NGC 2121 (2.9 Gyr) are from Kerber et al. (2007). [See the electronic edition of the Journal for a color version of this figure.]

(LMC) star clusters are overlaid with $\mathrm{BC} 03, \mathrm{M} 05$, and our computations in the $[\mathrm{Fe} / \mathrm{H}]$ versus $(B-V)_{0}($ bottom $)$ and $(V-K)_{0}$ (top) plots. LMC star clusters' integrated $(B-V)$ colors are from van den Bergh (1981). Their reddening and $(V-K)_{0}$ and SWB types are from Table 4 of Persson et al. (1983). The ages and metallicities of these star clusters are adopted from Table 2 of Pessev et al. (2006). We note, however, that recent measurements of higher metallicity LMC clusters $([\mathrm{Fe} / \mathrm{H}]>-1.0 \mathrm{dex})$ by Grocholski et al. (2006) show a very tight metallicity distribution (mean $[\mathrm{Fe} / \mathrm{H}]=-0.48, \sigma=0.09$ ). Among SWB types I-III, clusters younger than $100 \mathrm{Myr}$ are depicted with open triangles.

According to Frogel et al. (1990), the SWB type V star clusters in the LMC have bright AGBs with carbon stars (see also 


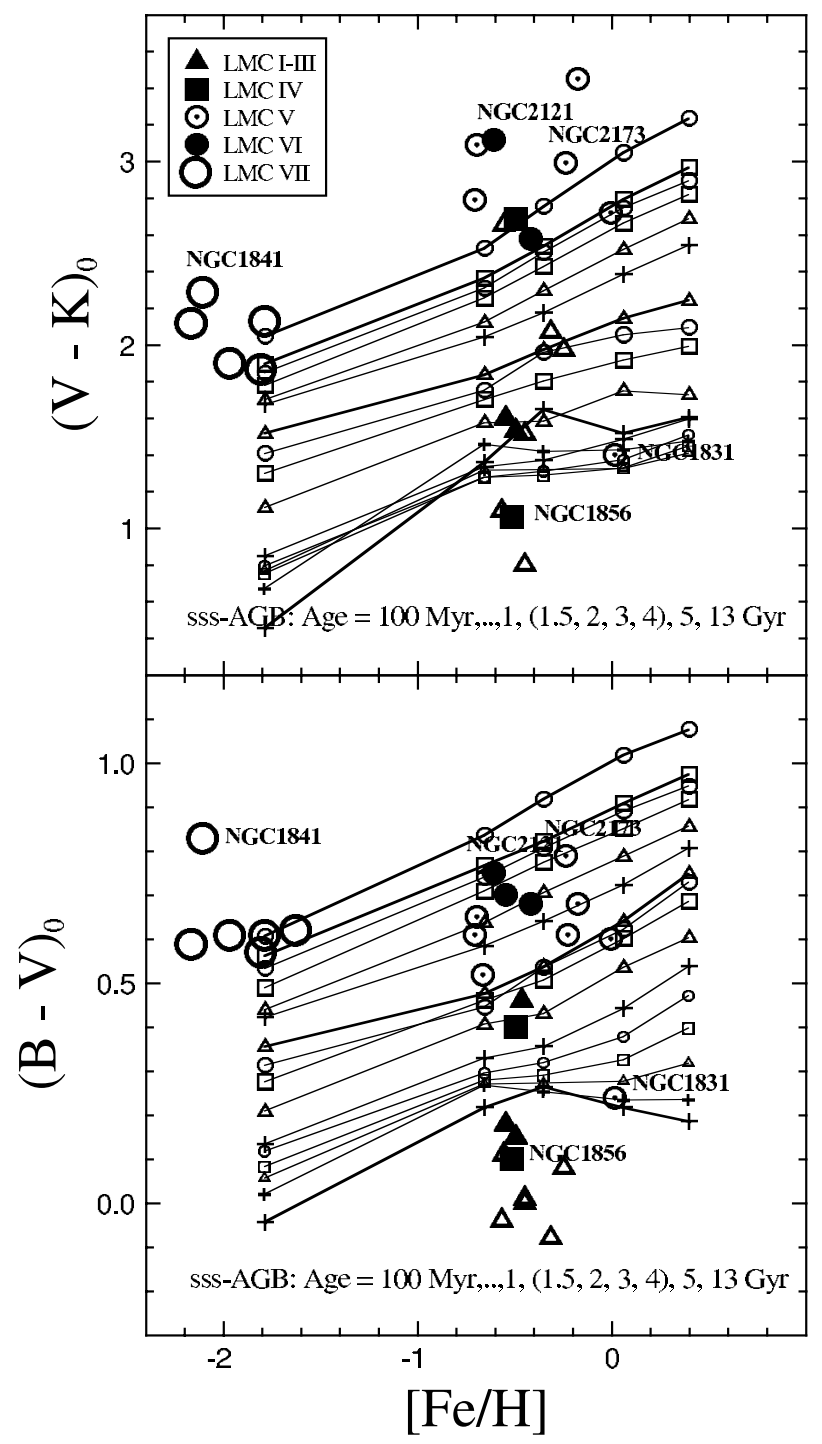

Fig. 10.- Same as Fig. 9, but our calculations with BaSTI-sss isochrones (without TPAGB phases) are compared with LMC star clusters. Recent age estimates of NGC 1856 (300 Myr), NGC 1831 (700 Myr), NGC 2173 (1.6 Gyr), and NGC 2121 $(2.9 \mathrm{Gyr})$ are from Kerber et al. (2007). Note that, contrary to $(B-V)$ colors, $(V-K)$ colors show some apparent mismatch between intermediate-age LMC star clusters and model predictions. [See the electronic edition of the Journal for a color version of this figure.]

Mucciarelli et al. [2006] for recent near-IR color-magnitude diagrams). Indeed, the $V-K$ colors of some of these LMC SWB type V star clusters (solar symbols in Figs. 8-12) are redder than the average trend. The observational scatter in $V-K$ color of LMC SWB types I-III star clusters should be understood by some other mechanism, such as stochastic effects (Santos \& Frogel 1997), but certainly not by metallicity (see also Goudfrooij et al. 2006). One of the old SWB VII clusters, NGC 1841, is also indicated in the plots because of its unusually red $(B-V)_{0}$ color. Its $(B-V)$ is 0.90 according to Table 3 of van den Bergh (1981) and its $E(B-V)$ is given as 0.07 in Table 4 of Persson et al. (1983). However, its peculiarity can be removed if we adopt 0.80 for its $(B-V)$ from Table 1 of Bica et al. (1996) and 0.18 for its $E(B-V)$ from Walker (1990).

Now we take a close look how different SSP models are compared with these LMC star clusters with a wide range of age and metallicity. First of all, LMC star clusters are overlaid on BC03

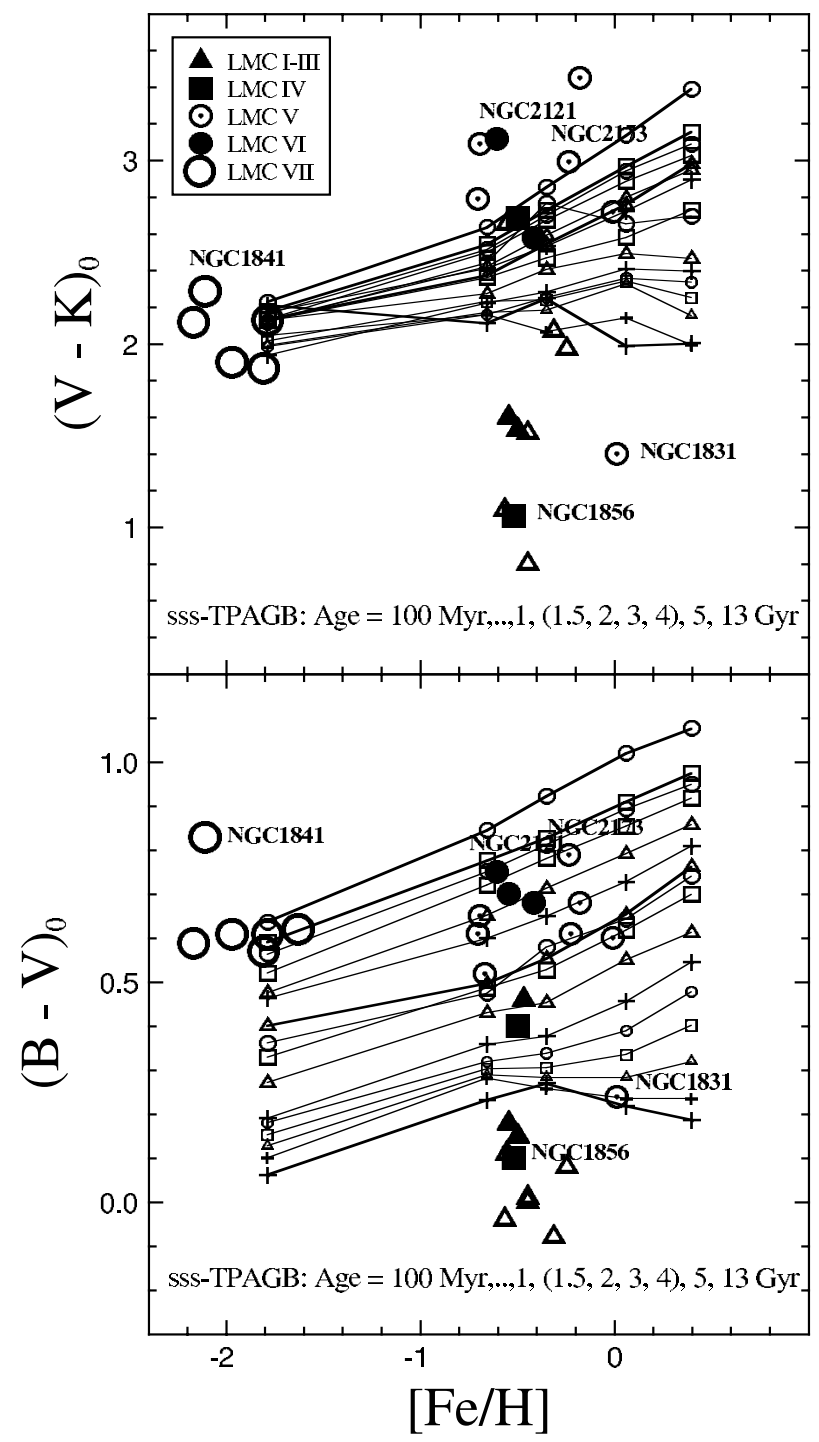

FIG. 11.- Same as Fig. 10, but our calculations with BaSTI-sss isochrones (with TPAGB phases) are compared with LMC star clusters. Recent age estimates of NGC 1856 (300 Myr), NGC 1831 (700 Myr), NGC 2173 (1.6 Gyr), and NGC 2121 (2.9 Gyr) are from Kerber et al. (2007). Note that the $(V-K)$ colors become significantly redder compared to Fig. 10 at younger ages because of the TPAGB phases although the mismatch between observations and models still lingers. [See the electronic edition of the Journal for a color version of this figure.]

models in Figure 8. Those four ages (100 Myr, 1, 5, $13 \mathrm{Gyr})$ that are shown in the previous color-color plots are delineated with lines with crosses, triangles, squares, and circles, respectively. To add detail, we also display model predictions at finer age spacings. At solar metallicity, eight $100 \mathrm{Myr}$ increments between $100 \mathrm{Myr}$ and $1 \mathrm{Gyr}$, and 1.5, 2, 3, and $4 \mathrm{Gyr}$ between 1 and $5 \mathrm{Gyr}$ are connected with dots. Both the $B-V$ and $V-K$ colors of BC03 models become monotonically redder with increasing age at given metallicity. Kerber et al. (2007) recently determined ages for four of the intermediate-age star clusters using deep HST colormagnitude diagrams (CMDs). Using Padova models, the ages of NGC 1856, NGC 1831, NGC 2173, and NGC 2121 were estimated as $300 \mathrm{Myr}, 700 \mathrm{Myr}, 1.6 \mathrm{Gyr}$, and $2.9 \mathrm{Gyr}$, respectively, and they are compared with models in Figures 8-12. It is noted that contrary to $B-V$ colors, $V-K$ colors show some evident mismatch between intermediate-age LMC star clusters and model predictions (see footnote 15). 


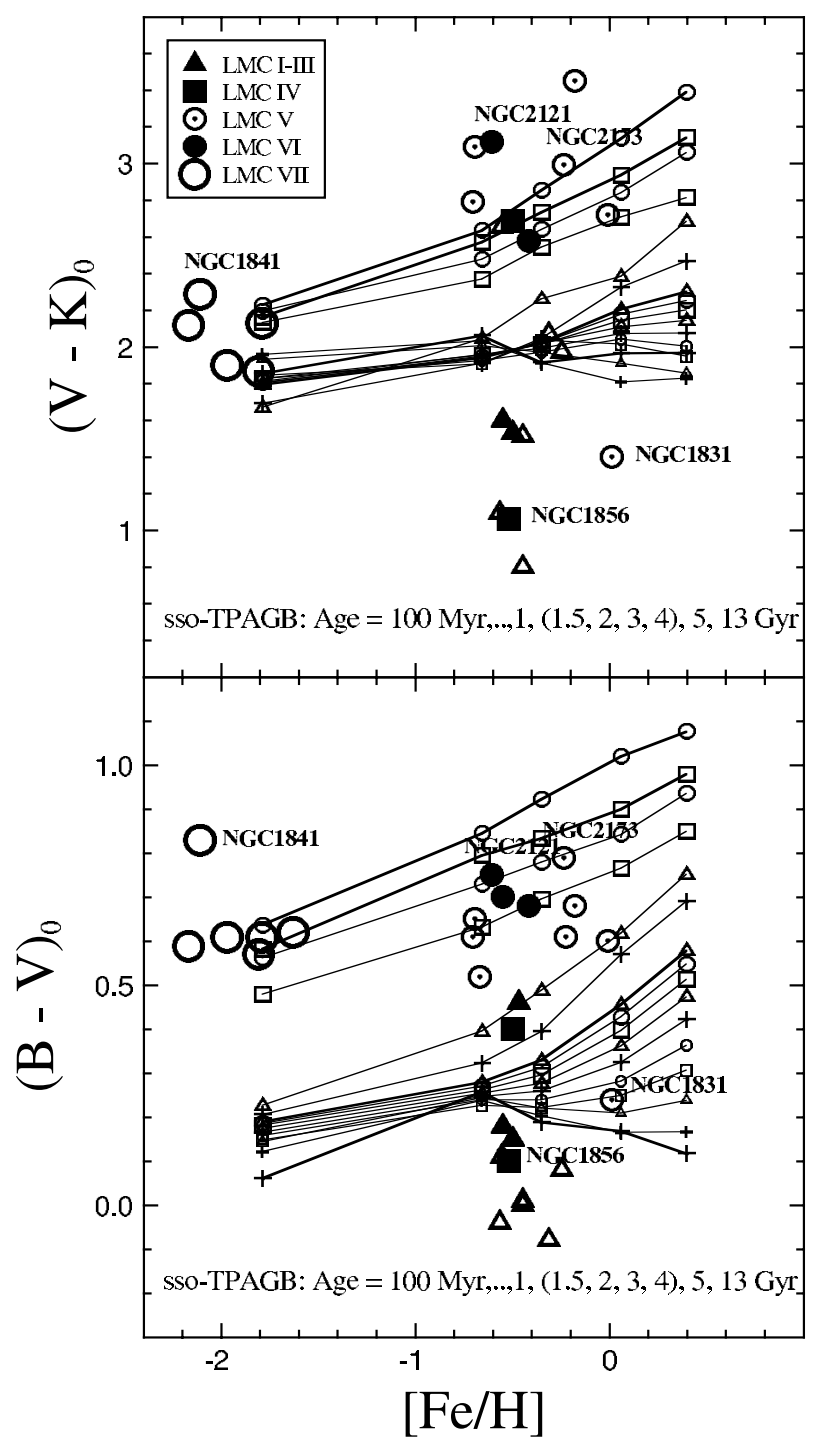

FIG. 12.- Same as Fig. 11, but our calculations with BaSTI-sso isochrones (with TPAGB phases) are compared with LMC star clusters. Note that both $(B-V)$ and $(V-K)$ colors become bluer compared to Fig. 11 at younger ages because of the convective core overshooting effects. Recent age estimates of NGC 1856 (300 Myr), NGC 1831 (700 Myr), NGC 2173 (1.6 Gyr), and NGC 2121 (2.9 Gyr) are from Kerber et al. (2007). Although this case should be the most realistic one, it does not ameliorate the mismatch between observations and models in $(V-K)$. [See the electronic edition of the Journal for a color version of this figure.]

Second, in Figure 9, M05 models are compared with LMC star clusters. Just like in the previous figure, four ages (100 Myr, 1, 5, $13 \mathrm{Gyr}$ ) that are shown in Figure 7 are delineated with thicker lines with crosses, triangles, squares, and circles, respectively. As we mentioned earlier, for a close examination, we now show every $100 \mathrm{Myr}$ increment between $100 \mathrm{Myr}$ and $1 \mathrm{Gyr}$, and 1.5, 2, 3, and 4 Gyr between 1 and $5 \mathrm{Gyr}$ from her models. We have used crosses, triangles, squares, and circles repeatedly for the description of increasing ages. While the $B-V$ colors become monotonically redder with increasing age at given metallicity, her $V-K$ colors show a sudden leap at 300 Myr because of the onset of the TPAGB. Note that M05 tuned her fuel consumption theorem parameters using the older age estimation of intermediate-age LMC star clusters. The exact epoch of onset will be satisfactorily constrained in the near future with a greater number of detailed deep CMDs of nearby young and intermediate-age star clusters

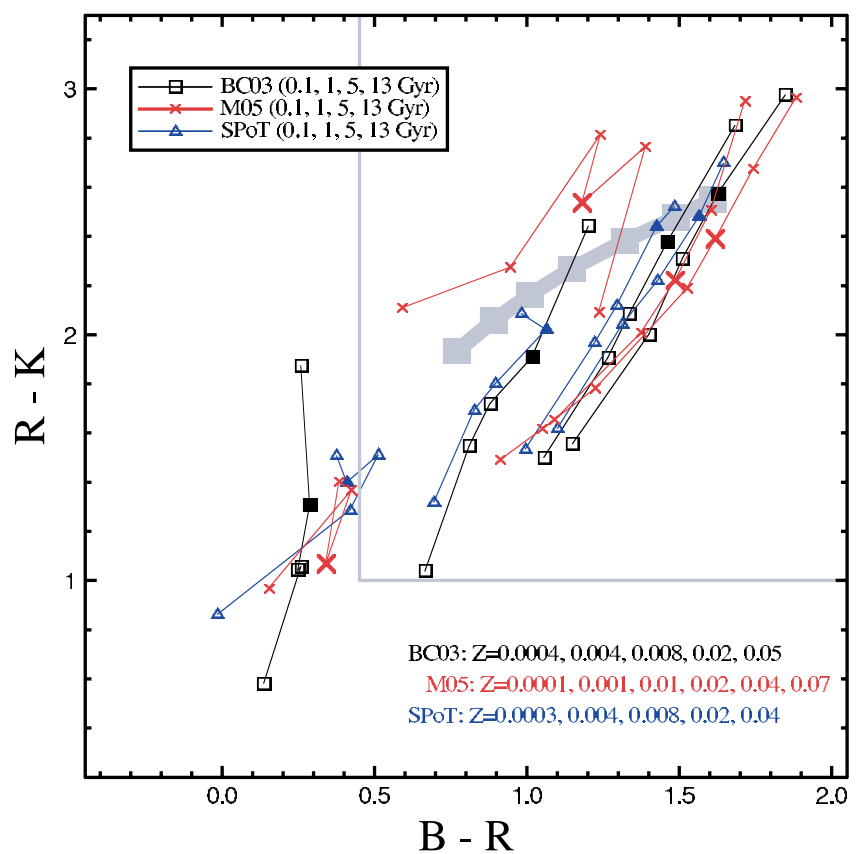

FIG. 13.-Similar to Figs. 6 and 7, but BC03, M05, and the SPoT simple stellar population models at $0.1,1,5$, and $13 \mathrm{Gyr}$ are compared in $(B-R)$ vs. $(R-K)$. BC03's solar metallicity composite models from Fig. 14 are shown here as the thick line with big filled squares (the seven average ages, $\langle A\rangle$ ) to compare the SSP and the CSP models. For the same purpose, the matching scales of Fig. 14 are delineated with gray straight lines. According to Fig. 11 of MacArthur et al. (2004), spiral galaxies with high rotational velocity mostly swarm around this thick line.

along with the help of improved stellar models (e.g., Kerber et al. 2007; Mucciarelli et al. 2007).

Finally, our calculations with BaSTI are presented in Figures $10-12$. Figure 10 shows our calculations employing BaSTI-sss isochrones with the exclusion of TPAGB phases, while Figure 11 illustrates those with TPAGB phases. It is seen that the $V-K$ colors become significantly redder at younger ages because of the TPAGB phases, whereas they do not affect $B-V$ colors much. The apparent mismatch in $V-K$ colors between our calculations and intermediate-age LMC star clusters lingers even with TPAGB phases. In Figure 12, we add convective core overshooting effects along with a TPAGB using BaSTI-sso isochrones. It is seen that both $B-V$ and $V-K$ colors become bluer compared to Figure 11 at younger ages because of the convective core overshooting effects. Although this case should be the most realistic one, it does not ameliorate the mismatch between observations and models at $V-K$ colors. It is believed, however, that all these discrepancies will be narrowed down with the improvement of stellar models (e.g., Marigo 2007).

Having studied the model differences and their matches against the observations in $B V K$ photometry, in Figure 13, we switch from $B-V$ versus $V-K$ to $B-R$ versus $R-K$, as Bell \& de Jong (2000) and MacArthur et al. (2004) presented their spiral galaxy data in these colors. Figure 13 is similar to Figures 6 and 7, but here $\mathrm{BC} 03, \mathrm{M} 05$, and SPoT SSP models at $0.1,1,5$, and $13 \mathrm{Gyr}$ are compared in the $B-R$ versus $R-K$ diagram. This is the basis of Figure 14, the composite model grids when the Figure 13 SSP models are convolved with exponentially declining SFHs. In addition, in Figure 13 BC03's solar metallicity composite models from Figure 14 are shown as big filled squares connected by a thick line (indicating the seven average ages, $\langle A\rangle$, in Fig. 14) to compare SSP and CSP models. According to Figure 11 of MacArthur et al. 


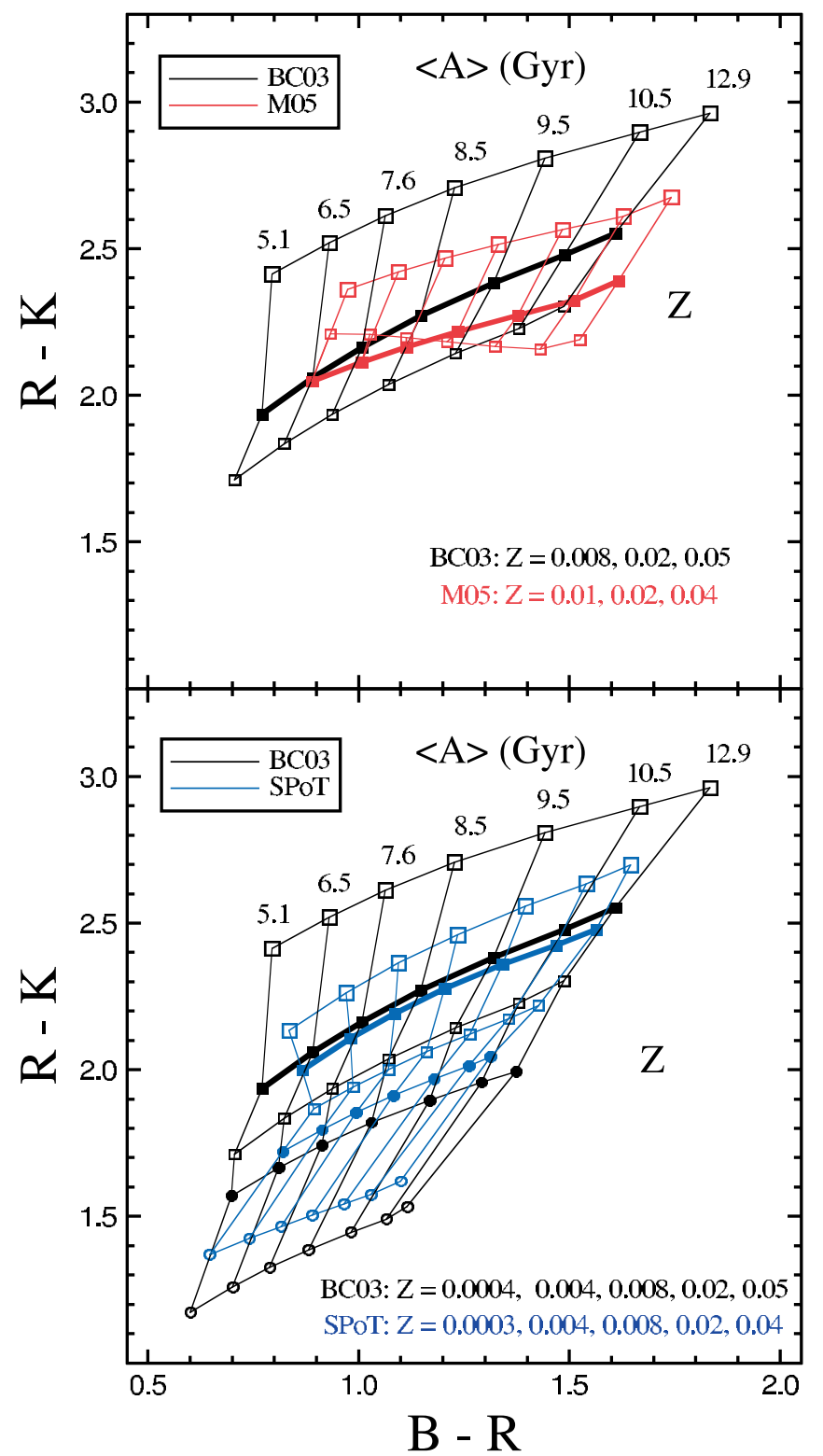

FIG. 14.- Three different groups' composite stellar population models are generated as identical SFHs are employed. BC03 (black lines) and M05 (red lines) are compared in the top panel and $\mathrm{BC} 03$ and SPoT (blue lines) are contrasted in the bottom panel. The same symbols are used for similar metallicities. To guide the eye, solar metallicities are depicted with thicker lines. Note also the compression of scale compared to the Fig. 13 SSP illustration.

(2004), spiral galaxies with high rotational velocity mostly swarm around this thick line.

\section{RESULTS AND DISCUSSION}

Composite stellar population models from three different groups (BC03, M05, and SPoT) are shown in Figure 14. BC03 (black lines) and M05 (red lines) are compared in the top panel and $\mathrm{BC} 03$ and SPoT (blue lines) are contrasted in the bottom panel. We have generated each model grid using identical star formation rates (SFRs). The simple exponential SFR that we use in this study is $\Psi_{\text {exp }}(t)$,

$$
\Psi_{\exp }(t)=\frac{c_{\exp }}{\tau} e^{-t / \tau}
$$

TABLE 1

Comparison of Average Ages at Solar Metallicity for BC03 Models

\begin{tabular}{|c|c|c|c|c|}
\hline$\tau^{\mathrm{a}}$ & $\langle A\rangle^{\mathrm{b}}$ & $\langle A\rangle_{\text {cutoff }^{\mathrm{c}}}$ & $\langle A\rangle_{V}{ }^{\mathrm{d}}$ & $\langle A\rangle_{K}{ }^{\mathrm{e}}$ \\
\hline-10 & 5.1 & 5.2 & 1.8 & 3.0 \\
\hline $500 \ldots \ldots \ldots \ldots \ldots \ldots \ldots \ldots \ldots \ldots$ & 6.5 & 6.5 & 2.6 & 4.2 \\
\hline $13 \ldots \ldots+\cdots$ & 7.6 & 7.6 & 3.5 & 5.4 \\
\hline 6.5 & 8.5 & 8.5 & 4.8 & 6.7 \\
\hline 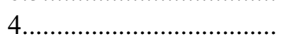 & 9.5 & 9.5 & 6.7 & 8.2 \\
\hline $2.6 \ldots \ldots \ldots$ & 10.5 & 10.5 & 9.1 & 9.8 \\
\hline 0.1 & 12.9 & 12.9 & 12.9 & 12.9 \\
\hline
\end{tabular}

${ }^{\text {a }}$ It may be useful to refer to the top portion of Fig. 4 of MacArthur et al. (2004) for the shape of star formation rate over ages with given $\tau$. A couple of cases of bursty SFHs are also demonstrated in Figs. 6 and 7 of MacArthur et al. (2004).

b Mass-weighted average age in Gyr.

${ }^{c}$ Mass-weighted average age in Gyr with $100 \mathrm{Myr}$ cutoff.

${ }^{d} V$ luminosity-weighted average age in Gyr.

${ }^{\mathrm{e}} K$ luminosity-weighted average age in Gyr.

where

$$
c_{\exp }=\frac{1}{1-e^{-A / \tau}}
$$

and $A=13 \mathrm{Gyr}$. The average age by which they are labeled is a mass-weighted average age and at a given $\tau$, computed as

$$
\langle A\rangle=A-\tau \frac{1-e^{-A / \tau}(1+A / \tau)}{1-e^{-A / \tau}} .
$$

For example, a constant star formation rate over 13 Gyr yields an average age of 6.5 Gyr. If weighting by luminosity rather than mass, one arrives at much younger ages. For comparison, we list $V$ - and $K$-band luminosity-weighted ages at solar metallicity for BC03 models along with the $\tau$ and mass-weighted ages in Table 1 . Our mass-weighted age is actually the $\langle A\rangle_{\text {cutoff }}$ that appears in the third column of Table 1. This is because we have considered the most recent star formation to be truncated at $100 \mathrm{Myr}$ in this study. ${ }^{17}$ In Table 1, the luminosity-weighted ages are indeed much younger than the mass-weighted ages because young stellar components dominate the light in the composite populations, as can be seen from Figure 13 when SSP ages are compared to the CSP ages.

The models illustrated in Figure 14 are clearly different from one another, although they seem to agree to a certain degree at solar metallicity (delineated with thick lines to guide the eye). The differences arise from the original dissimilarities of simple stellar populations. In fact, the averaging process for the $\tau$ model CSPs smooths over many of the angularities present in the SSP grids to yield something more regular. It is also worthwhile to note that these composite grids are built at fixed metallicity, without any chemical evolution. The behavior of the CSP models echoes that of the SSP ones, and especially that of the younger components, because younger subpopulations are brighter and tend to dominate the light. In the case of M05 CSP models, the iso-metallicity lines of $Z=0.01$ and 0.02 even cross over, echoing their corresponding SSP models at $100 \mathrm{Myr}$ and at $1 \mathrm{Gyr}$. The SPoT's CSP grids

\footnotetext{
${ }^{17}$ Besides the reason that we noted in $\S 2$, it is seen in Table 1 that only the $\langle A\rangle=5.1 \mathrm{Gyr}$ case is affected by the $100 \mathrm{Myr}$ minimum age, and the effect is negligible. Although there is ongoing star formation $(t<100 \mathrm{Myr})$ in spiral galaxies, that mass fraction is not usually a major component (e.g., Hammer et al. 2005).
} 


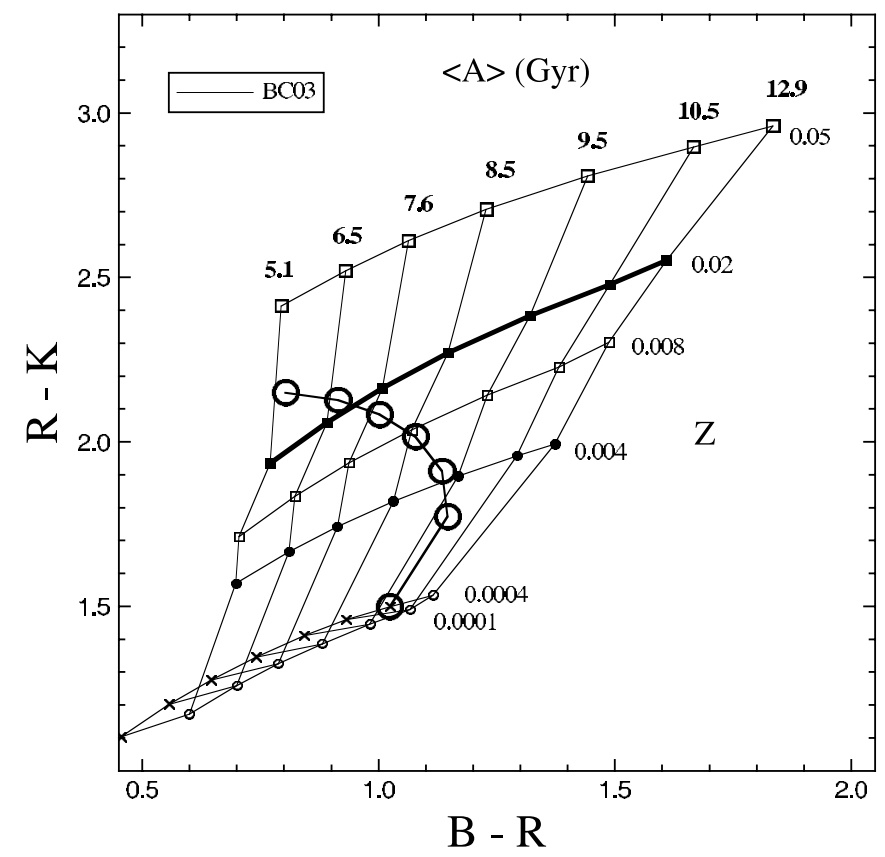

FIG. 15.- Similar to Fig. 14, with the BC03 models repeated (black lines), but a chemical enrichment scheme with a monotonic age-metallicity relation is incorporated in the $\mathrm{BC} 03$ composite stellar population models at given average ages (circles; see text). [See the electronic edition of the Journal for a color version of this figure.]

are much more compact in $R-K$ colors compared to $\mathrm{BC} 03$, both at the metal-poor and at the metal-rich side, also echoing their original SSP models.

We have used the same symbols for similar metallicities in Figure 14 in order to make the comparisons easy. The highest metallicities of three models are almost the same: the highest metallicity in $\mathrm{BC} 03$ is $Z=0.05$ with $Y=0.352$, while the two other are $Z=0.04$ with $Y=0.303$ (SPoT) and 0.340 (M05). The thick lines in Figure 14 are solar iso-metallicity lines for each CSP model. We hope that the fact that different models become similar at this rather critical metallicity is a robust result and not a coincidence. Reading from Figure 14, a galaxy with colors $B-R=0.97$ and $R-K=2.37$ would, in the M05 $\tau$ models, have an age of $5.1 \mathrm{Gyr}$ and $Z=0.04$, or $[\mathrm{Fe} / \mathrm{H}]=+0.3$ where $[\alpha / \mathrm{Fe}]=0$. The same galaxy would have $($ age $, Z,[\mathrm{Fe} / \mathrm{H}])=$ $(7.0,0.037,0.27)$ according to $\mathrm{BC} 03$, or $(6.7,0.052,0.41)$ according to SPoT. Taking another galaxy as an example, with $B-R=1.61$ and $R-K=2.39$, M05 would indicate (age, $Z,[\mathrm{Fe} / \mathrm{H}])=(13,0.02,0.00)$, while BC03 indicates $(15,0.008$, $-0.40)$ and SPoT indicates $(16,0.012,-0.22)$. The uncertainties appear to be in line with those tabulated in Charlot et al. (1996), about $30 \%$ in age. The scatter in abundance may be a bit larger than the 0.1 dex predicted by Charlot et al. (1996). In terms of relative $\Delta Z / \Delta$ age measurements, such as would be applied to spatial gradient data, Figure 14 yields similar percentage scatter.

Notwithstanding the incorporation of star formation history using the exponentially declining $\tau$ models, Figure 14 is still far from a realistic description of stellar populations of spiral galaxies. This is because even models like those in Figure 14 do not yet include any form of chemical evolution because they are built at fixed metallicity. In Figure 15, we experiment with the same exponential SFR that we have adopted in Figure 14, but with one very simple and useful case of chemical enhancement where metallicity is varied along with age. For this experiment, $Z=0.0001$ is assigned for the stars that formed at 13 Gyr ago, $Z=0.0004$ for the stars formed at 11 and 12 Gyr ago, $Z=0.004$ for the stars formed at 9 and 10 Gyr ago, $Z=0.008$ for the stars formed at 7 and $8 \mathrm{Gyr}$ ago, $Z=0.02$ for the stars formed at 2, 3, 4, 5 and 6 Gyr ago, and $Z=0.05$ is given for the stars that formed at 0.1 and 1 Gyr. These models are marked by big circles in Figure 15, one for each mass-weighted average age, $\langle A\rangle$. In this case with strong age-metallicity relation, the CSP models with older average ages become considerably bluer in $R-K$ because of their (assumed) low metallicity, as most star formation happens at very early cosmic time. Models with younger average ages, on the other hand, become progressively redder because recent star formation has high metallicity.

These models are clearly far from the observations of spiral galaxies (roughly located near the thick line in Fig. 15, according to Fig. 11 of MacArthur et al. 2004) suggesting that this toy model with a strong age-metallicity relation is incorrect. However, the toy model serves to illustrate that a general trend caused by any chemical evolution scheme with an age-metallicity relation will cause a tilt in the model grids. The toy model is an extreme case, but almost any chemical evolution scheme will share the characteristic that star formation starts at low metallicity at early times and works toward high metallicity at late times. This will, in every case, induce a bias in the CSP color-color plots. Clearly, future work should include chemical evolution if coherent age results are the goal.

To summarize our results, the youngest populations in composite stellar population models dominate the light and act to defeat the otherwise universal age-metallicity degeneracy in color-color diagrams that employ optical and near-IR photometry. But scatter from model to model is large at simple stellar population ages $t<2$ Gyr. The dominant uncertainties in stellar population models arise from the convective core overshoot and the TPAGB phase, but helium abundance may play a significant role at higher metallicities. The various models show notable differences that result in considerably dissimilar composite grids in integrated light. These results suggest that the interpretation of rest-frame nearIR photometry is severely hampered by model uncertainties and therefore that the determination of ages and metallicities of spiral galaxies from optical and near-IR photometry may be harder than previously thought. Another source of cosmic variation could be introduced by the character of the star formation, whether it is smoothly varying like the models or characterized by bursts. Burstiness will cause a partial reversion to the SSP case, which has larger model-to-model scatter than the CSP models. Furthermore, it is emphasized that the current composite stellar population models do need some implementation of chemical enrichment histories for full interpretation of the observational data.

It is a great pleasure to thank Lauren MacArthur, Santi Cassisi, Gustavo Bruzual, Claudia Maraston, Mustapha Mouhcine, Gabriella Raimondo, Alessandro Bressan, Roelof de Jong, Aaron Dotter, and John Blakeslee for many helpful discussions. We also thank the anonymous referee for her/his thoughtful comments and insightful suggestions, which improved this paper greatly. Support for this work was provided by the NSF through grant AST-0307487, the New Standard Stellar Population Models (NSSPM) project. 


\section{REFERENCES}

Bell, E. F., \& de Jong, R. S. 2000, MNRAS, 312, 497

Bertelli, G., Bressan, A., Chiosi, C., Fagotto, F., \& Nasi, E. 1994, A\&AS, 106, 275

Bica, E., Claria, J. J., Dottori, H., Santos, J. F. C., Jr., \& Piatti, A. E. 1996, ApJS, 102, 57

Blanco, B. M., Blanco, V. M., \& McCarthy, M. F. 1978, Nature, 271, 638

Bruzual, G. 2007, preprint (astro-ph/0702091)

Bruzual, G., \& Charlot, S. 2003, MNRAS, 344, 1000 (BC03)

Chabrier, G. 2003, PASP, 115, 763

Charlot, S., Worthey, G., \& Bressan, A. 1996, ApJ, 457, 625

Cohen, J. G., Persson, S. E., Elias, J. H., \& Frogel, J. A. 1981, ApJ, 249, 481

Cordier, D., Pietrinferni, A., Cassisi, S., \& Salaris, M. 2007, AJ, 133, 468

de Jong, R. S. 1996, A\&A, 313, 377

Demarque, P., Woo, J.-H., Kim, Y.-C., \& Yi, S. K. 2004, ApJS, 155, 667

Deupree, R. G. 1998, ApJ, 499, 340 2000, ApJ, 543, 395

Ferraro, F. R., Origlia, L., Testa, V., \& Maraston, C. 2004, ApJ, 608, 772

Frogel, J. A., Mould, J., \& Blanco, V. M. 1990, ApJ, 352, 96

Frogel, J. A., Persson, S. E. \& Cohen, J. G. 1980, ApJ, 239, 495

Gallart, C., Zoccali, M., \& Aparicio, A. 2005, ARA\&A, 43, 387

Girardi, L., Bressan, A., Bertelli, G., \& Chiosi, C. 2000, A\&AS, 141, 371

Goudfrooij, P., Gilmore, D., Kissler-Patig, M., \& Maraston, C. 2006, MNRAS, 369,697

Grocholski, A. J., Cole, A. A., Sarajedini, A., Geisler, D., \& Smith, V. V. 2006, AJ, 132, 1630

Groenewegen, M. A. T., \& de Jong, T. 1993, A\&A, 267, 410

Groenewegen, M. A. T., Smith, C. H., Wood, P. R., Omont, A., \& Fujiyoshi, T. 1995, ApJ, 449, L119

Hammer, F., Flores, H., Elbaz, D., Zheng, X. Z., Liang, Y. C., \& Cesarsky, C. 2005, A\&A, 430, 115

James, P. A., Salaris, M., Davies, J. I., Phillipps, S., \& Cassisi, S. 2006, MNRAS, 367, 339

Kerber, L. O., Santiago, B. X., \& Brocato, E. 2007, A\&A, 462, 139

Kroupa, P. 2002, Science, 295, 82

Kundu, A., et al. 2005, ApJ, 634, L41

Larsen, S. S., Brodie, J. P., \& Strader, J. 2005, A\&A, 443, 413

Lee, H.-C., Gibson, B. K., Flynn, C., Kawata, D., \& Beasley, M. A. 2004, MNRAS, 353, 113
Lee, H.-C., Lee, Y.-W., \& Gibson, B. K. 2002, AJ, 124, 2664

Liu, M. C., Charlot, S., \& Graham, J. R. 2000, ApJ, 543, 644

MacArthur, L. A., Courteau, S., Bell, E. F., \& Holtzman, J. A. 2004, ApJS, 152 , 175

Maraston, C. 2005, MNRAS, 362, 799 (M05)

Maraston, C., Daddi, E., Renzini, A., Cimatti, A., Dickinson, M., Papovich, C., Pasquali, A., \& Pirzkal, N. 2006, ApJ, 652, 85

Marigo, P. 2007, preprint (astro-ph/0701536)

Marigo, P., Girardi, L., \& Chiosi, C. 2003, A\&A, 403, 225

Meynet, G., Eggenberger, P., \& Maeder, A. 2007, preprint (astro-ph/0702515)

Meynet, G., \& Maeder, A. 2000, A\&A, 361, 101

Mould, J., \& Aaronson, M. 1979, ApJ, 232, 421 1980, ApJ, 240, 464

Mucciarelli, A., Ferraro, F. R., Origlia, L., \& Fusi Pecci, F. 2007, AJ, 133, 2053

Mucciarelli, A., Origlia, L., Ferraro, F. R., Maraston, C., \& Testa, V. 2006, ApJ, 646, 939

Paczyński, B. 1971, Acta Astron., 21, 417

Persson, S. E., Aaronson, M., Cohen, J. G., Frogel, J. A., \& Matthews, K. 1983, ApJ, 266, 105

Pessev, P. M., Goudfrooij, P., Puzia, T. H., \& Chandar, R. 2006, AJ, 132, 781

Pietrinferni, A., Cassisi, S., Salaris, M., \& Castelli, F. 2004, ApJ, 612, 168

Puzia, T. H., Zepf, S. E., Kissler-Patig, M., Hilker, M., Minniti, D., \& Goudfrooij, P. 2002, A\&A, 391, 453

Raimondo, G., Brocato, E., Cantiello, M., \& Capaccioli, M. 2005, AJ, 130, 2625

Salasnich, B., Girardi, L., Weiss, A., \& Chiosi, C. 2000, A\&A, 361, 1023

Salpeter, E. E. 1955, ApJ, 121, 161

Santos, J. F. C., Jr., \& Frogel, J. A. 1997, ApJ, 479, 764

Searle, L., Wilkinson, A., \& Bagnuolo, W. G. 1980, ApJ, 239, 803 (SWB)

VandenBerg, D. A., Bergbusch, P. A., \& Dowler, P. D. 2006, ApJS, 162, 375 van den Bergh, S. 1981, A\&AS, 46, 79

Vassiliadis, E., \& Wood, P. R. 1993, ApJ, 413, 641

Walker, A. R. 1990, AJ, 100, 1532

Worthey, G. 1994, ApJS, 95, 107

Yi, S., Demarque, P., Kim, Y.-C., Lee, Y.-W., Ree, C. H., Lejeune, T., \& Barnes, S. 2001, ApJS, 136, 417 